\title{
Psychoactive substance use and the risk of motor vehicle accidents
}

\author{
K.L.L. Movig a,b,1, M.P.M. Mathijssen ${ }^{\text {c }}$, P.H.A. Nagel ${ }^{\mathrm{d}}{ }^{\text {, T. }}$ van Egmond ${ }^{\mathrm{d}}$, \\ J.J. de Gier ${ }^{b}$, H.G.M. Leufkens ${ }^{b}$, A.C.G. Egberts ${ }^{a, b, *}$ \\ ${ }^{a}$ Hospital Pharmacy Midden-Brabant, St. Elisabeth Hospital and TweeSteden hospital, Tilburg, The Netherlands \\ ${ }^{\mathrm{b}}$ Department of Pharmacoepidemiology and Pharmacotherapy, Utrecht Institute for Pharmaceutical Sciences (UIPS), \\ Utrecht University, Sorbonnelaan 16, P.O. Box 80082, 3508 TB Utrecht, The Netherlands \\ c SWOV Institute for Road Safety Research, Leidschendam, The Netherlands \\ d Department of Surgery, St. Elisabeth Hospital, Tilburg, The Netherlands
}

Received 12 November 2002; received in revised form 12 May 2003; accepted 30 May 2003

\begin{abstract}
The driving performance is easily impaired as a consequence of the use of alcohol and/or licit and illicit drugs. However, the role of drugs other than alcohol in motor vehicle accidents has not been well established. The objective of this study was to estimate the association between psychoactive drug use and motor vehicle accidents requiring hospitalisation.

A prospective observational case-control study was conducted in the Tilburg region of The Netherlands from May 2000 to August 2001. Cases were car or van drivers involved in road crashes needing hospitalisation. Demographic and trauma related data was collected from hospital and ambulance records. Urine and/or blood samples were collected on admission.

Controls were drivers recruited at random while driving on public roads. Sampling was conducted by researchers, in close collaboration with the Tilburg police, covering different days of the week and times of the day. Respondents were interviewed and asked for a urine sample. If no urine sample could be collected, a blood sample was requested.

All blood and urine samples were tested for alcohol and a number of licit and illicit drugs. The main outcome measures were odds ratios (OR) for injury crash associated with single or multiple use of several drugs by drivers.

The risk for road trauma was increased for single use of benzodiazepines (adjusted OR 5.1 (95\% Cl: 1.8-14.0)) and alcohol (blood alcohol concentrations of $0.50-0.79 \mathrm{~g} / \mathrm{l}$, adjusted OR $5.5(95 \% \mathrm{Cl}$ : $1.3-23.2)$ and $\geq 0.8 \mathrm{~g} / \mathrm{l}$, adjusted OR 15.5 (95\% Cl: 7.1-33.9)). High relative risks were estimated for drivers using combinations of drugs (adjusted OR 6.1 (95\% Cl: 2.6-14.1)) and those using a combination of drugs and alcohol (OR 112.2 (95\% Cl: 14.1-892)). Increased risks, although not statistically significantly, were assessed for drivers using amphetamines, cocaine, or opiates. No increased risk for road trauma was found for drivers exposed to cannabis.

The study concludes that drug use, especially alcohol, benzodiazepines and multiple drug use and drug-alcohol combinations, among vehicle drivers increases the risk for a road trauma accident requiring hospitalisation.
\end{abstract}

(C) 2003 Elsevier Ltd. All rights reserved.

Keywords: Traffic accidents; Injured drivers; Alcohol; Drugs; Medicines; Case-control study

\section{Introduction}

The Grand Rapids Study convincingly showed that driving under influence of alcohol is an important risk factor for traffic accidents (Borkenstein et al., 1974). The role of drugs other than alcohol in motor vehicle accidents, however, has not been well established. Many medicines (prescription or over-the-counter) and illicit drugs affect the nervous system (Ramaekers, 1998). Driving under the influence of drugs

\footnotetext{
* Corresponding author. Tel.: +31-30-2537323; fax: +31-30-2539166. E-mail address: a.c.g.egberts@pharm.uu.nl (A.C.G. Egberts).

${ }^{1}$ Present address: Department of Clinical Pharmacy, Medical Spectrum Twente Hospital group, Enschede, The Netherlands.
}

other than alcohol is considered to be an increasing cause of traffic accidents worldwide (Morland, 2000).

Several classes of drugs, including amphetamines, antihistamines, cannabis, hypnotics, tranquillizers and tricyclic antidepressants, have been shown to impair driving skills in laboratory tests and driver-simulation studies (O'Hanlon and Volkerts, 1986; Smiley, 1987; Robbe, 1998). These studies are restricted in various ways, e.g. with regard to subject selection and divergent behaviour or sample size. Their generalization to a 'real' traffic situation is debated. Many observational studies have shown that drug use as determined by biological sampling among drivers is prevalent and increasing (Gjerde et al., 1993; Soderstrom et al., 1997; Morland, 2000). Several studies tried to provide 
accident risk estimates related to drug use by linking drug prescription records to hospital admission records involving motor vehicle accidents, police reports, or health insurance records (Skegg et al., 1979; Ray et al., 1992; Leveille et al., 1994; Neutel, 1995; Hemmelgarn et al., 1997; Barbone et al., 1998; McGwin et al., 2000). In addition, a few studies have been conducted in which non-trauma patients were included as controls (Honkanen et al., 1980; Marquet et al., 1998). These studies provided risk estimates, but a limitation is that the control groups were not taken from moving traffic.

The objective of our study was to estimate the association between the use of various psychoactive substances and serious traffic injuries needing hospitalisation by comparing exposure frequencies (prevalences) of these substances in injured and non-accident involved drivers.

\section{Methods}

\subsection{Setting}

The study was conducted in co-operation with a large general teaching hospital (St. Elisabeth Hospital) and the police department located in the Dutch city of Tilburg, covering a population of approximately 350,000 persons. The hospital has all the facilities comparable with a highly equipped trauma centre. The source populations of our study were injured and non-accident involved drivers throughout the period from May 2000 until August 2001. The hospital's Medical Ethics Committee approved the study protocol and informed consent was obtained for the use of medical records from patients or their relatives.

\subsection{Study design}

A prospective case-control study was conducted to assess the relationship between drug use and trauma injuries requiring hospitalisation caused by motor vehicle accidents. Only injured car or van drivers were included in the study.

\subsection{Selection of cases}

All injured car or van drivers who were admitted to the emergency room of the hospital were prospectively identified. Demographic, accident and injury characteristics were obtained from medical and ambulance records, and the emergency's department electronic medical data system (Vles et al., 2000). The physicians at the emergency department were trained to fill in a detailed questionnaire about the crash circumstances. The severity of injuries was graded according to the Injury Severity Scale (ISS) (Baker et al., 1974). Urine and/or blood samples were taken directly in the emergency room.

\subsection{Selection of controls}

Control patients were randomly selected from moving traffic during 20 roadside survey sessions. In order to be able to construct a representative control sample, the week was systematically divided into $6 \mathrm{~h}$ periods. Each survey session covered a $6 \mathrm{~h}$ period because of work-time reasons. The survey was conducted among a representative sample of locations along main roads in the Tilburg police district, which covers the area of the St. Elisabeth Hospital. Frequent change of location was intended to minimize the predictability of the surveys with respect to time and place.

The police stopped cars and the drivers were asked to cooperate on a voluntary basis. The survey sessions were combined with normal police enforcement activities regarding drink-driving. Respondents were interviewed on their drug and medicine use and subsequently requested to produce a urine specimen. If they were not able or willing to urinate they were requested to deliver a blood specimen. A trained research nurse performed the vena-puncture. Subjects who delivered a urine or blood specimen were compensated by a small amount of 10 Dutch guilders (approximately $€ 5$ ). The interview and specimen sampling took place in a specially equipped mobile research unit with a private toilet. After the interview and specimen sampling, all subjects were breath tested for alcohol by a police officer, using a Dräger Alcotest 7410 Plus screening device. The breath test was compulsory for all drivers who were stopped. Data collection also comprised date and time of selection, gender and age of the driver, and signs of intoxication.

\subsection{Drug exposure assessment}

Both cases and controls were tested for the presence of alcohol, amphetamines, barbiturates, benzodiazepines, cannabis, methadone, opiates, and tricyclic antidepressants.

Urine samples were screened at the Dutch Laboratory for Drugs Doping, Tilburg, The Netherlands. Screening was performed by enzyme multiplied immunoassay technique (EMIT $^{\circledR}$ II Plus). For benzodiazepines, a special high sensitivity protocol was used with on-line deglucuronidation. The risk for false-positive results was avoided by confirmation analysis of the positive urine samples (amphetamines and opiates) with appropriate gas chromatography mass spectrometry (GC-MS) techniques.

Drug screening in serum was performed by The Netherlands Forensic Institute (NFI), Rijswijk, The Netherlands. Opiates and cannabis screening was performed by Cozart ${ }^{\circledR}$ enzyme immunoassay (EIA). Confirmation was performed using GC-MS. For the other drugs and pharmaceuticals, a high-performance liquid chromatography (HPLC) method was used, which was developed by NFI.

Concerning the cases medical and ambulance records were examined to control for drugs administered during transport and at the emergency department. When urine or blood specimens were positive for drugs given during 
transport, or in the emergency room before sampling, the specimens were considered to be negative.

\subsection{Potential confounders}

The following covariates were studied as possible confounding factors: age, gender, blood alcohol concentration, concomitant drug exposure, season, and time of day. Time of day was categorised as day (10 a.m.-10 p.m.) or night (10 p.m.-10 a.m.).

\subsection{Statistical analysis}

For both cases and controls, the baseline characteristics were determined. An ANOVA/Student's $t$-test was performed to assess the significance of differences in the mean of continuous variables between cases and controls. Differences in proportions of categorical variables were tested for significance by a chi-square $\left(\chi^{2}\right)$ test. To estimate the association between drug use, and any other potential risk factor and road trauma requiring hospitalisation adjusted odds ratios $(\mathrm{OR})$ with $95 \%$ confidence intervals $(95 \% \mathrm{Cl})$ were calculated using multivariate unconditional logistic regression (Hosmer and Lemeshow, 1989). All potential confounding factors were entered in the logistic model. In this model the odds ratio can be interpreted as a relative risk. Unadjusted ORs are not presented because this study was not designed to determine a causal relationship for each single drug separately. Data were analysed using SPSS 10.0 statistical software.

\section{Results}

We were able to identify and include 110 injured motorists. The relatives of two deceased patients refused consent and their data were excluded from analysis. The mean ISS for the case patients was 14 (range 1-75) of the 1029 controls, $816(79.3 \%)$ drivers complied to participate. In the case group $39 \%$ of the specimens consisted out of urine compared to $85 \%$ in the controls, respectively. Overall, $74 \%$ of the study subjects were men. The mean age was 38.6 years. Control subjects were older than case patients (39.1 versus 34.6, $P=0.002)$.

Table 1 shows the distribution of demographic variables and of the frequencies of drugs and alcohol use. Male patients were more frequently exposed to cannabis and cocaine. Drugs and/or alcohol were more frequently detected in injured drivers than in non-accident involved drivers.

Forty percent of all case patients were positive for one or more drugs and/or alcohol in comparison with $14 \%$ of the controls (Fig. 1). Eight percent of all case patients were both exposed to (multiple) drugs and alcohol. Only one control subject had used a drug-alcohol combination.

The use of benzodiazepines (adjusted OR 5.1 (95\% Cl: 1.8-14.0)) and alcohol was significantly associated with road
Table 1

Characteristics of cases and controls

\begin{tabular}{|c|c|c|}
\hline Characteristics & $\begin{array}{l}\text { Cases, } \\
N=110(\%)\end{array}$ & $\begin{array}{l}\text { Controls, } \\
N=816(\%)\end{array}$ \\
\hline \multicolumn{3}{|l|}{ Gender } \\
\hline Male & $81(74)$ & $602(74)$ \\
\hline Female & $29(26)$ & $214(26)$ \\
\hline \multicolumn{3}{|l|}{ Age group (year) } \\
\hline $18-25$ & $31(28)$ & 144 (18) \\
\hline $25-34$ & $35(32)$ & $228(28)$ \\
\hline $35-49$ & $28(26)$ & 237 (29) \\
\hline$\geq 50$ & $16(14)$ & $207(25)$ \\
\hline \multicolumn{3}{|l|}{ Season } \\
\hline January-March & $25(23)$ & $269(33)$ \\
\hline April-June & $21(19)$ & $172(21)$ \\
\hline July-September & $37(34)$ & 147 (18) \\
\hline October-December & $27(25)$ & $228(28)$ \\
\hline \multicolumn{3}{|l|}{ Time of day } \\
\hline 10 a.m.-10 p.m. & $54(49)$ & $496(61)$ \\
\hline 10 p.m.-10 a.m. & $56(51)$ & $320(39)$ \\
\hline \multicolumn{3}{|l|}{ Drugs and alcohol } \\
\hline Amphetamines & $7(6)$ & $13(2)$ \\
\hline Barbiturates & $2(2)$ & $0(0)$ \\
\hline Benzodiazepines & $11(10)$ & $12(1.5)$ \\
\hline \multicolumn{3}{|c|}{ Blood alcohol concentration $(\mathrm{g} / \mathrm{l})$} \\
\hline$<0.5$ & $84(76)$ & $796(97)$ \\
\hline $0.5-0.79$ & $3(3)$ & $7(1)$ \\
\hline$\geq 0.8$ & $23(21)$ & $13(2)$ \\
\hline Cannabis & $13(12)$ & $49(6)$ \\
\hline Cocaine & $10(9)$ & $16(2)$ \\
\hline Methadone & $1(1)$ & $0(0)$ \\
\hline Opiates & $8(7)$ & $20(3)$ \\
\hline Tricyclic antidepressants & $1(1)$ & $4(0.5)$ \\
\hline
\end{tabular}

accidents (Table 2). Drivers with a blood alcohol concentration $(\mathrm{BAC}) \geq 0.8 \mathrm{~g} / \mathrm{l}$ were at the highest risk (adjusted OR 15.5 (95\% Cl: 7.1-33.9)). Users of amphetamines, cocaine, and opiates had a twofold increased risk, however, not statistically significant. No association was found for cannabis and tricyclic antidepressants, and no estimates could be calculated with regard to methadone and barbiturates.

A sixfold increased risk (adjusted OR $6.1(95 \% \mathrm{Cl}$ : 2.60-14.10)) was found for the concurrent use of two, or more drugs compared with no drug use. This effect was even more pronounced in patients concomitantly exposed to alcohol and one or more drugs, which showed the highest risk for road accidents (adjusted OR $112.2(95 \% \mathrm{Cl}$ : 14.1-892.9)).

\section{Discussion}

This study indicates that use of alcohol, amphetamines, benzodiazepines, cocaine, and opiates places drivers at increased risk for motor vehicle accidents requiring hospitalisation. Users of drug-drug combinations were at a sixfold increased risk. Vehicle drivers who were exposed 


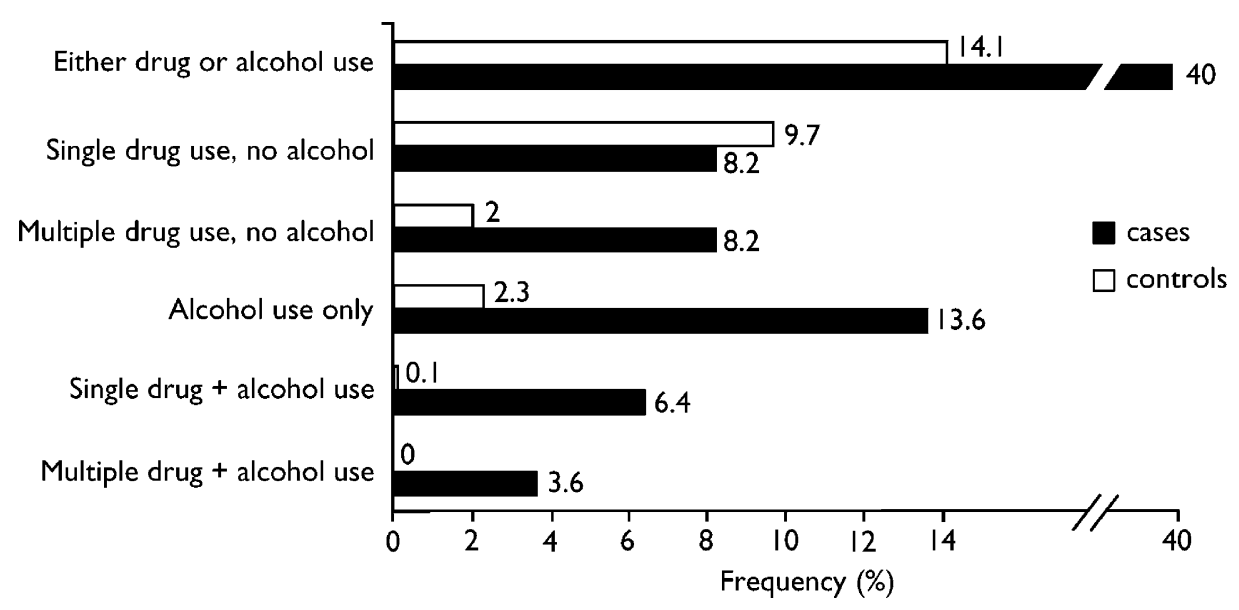

Fig. 1. Use of drugs and or alcohol $(\geq 0.5 \mathrm{~g} / \mathrm{l})$ for cases and controls.

to drug-alcohol combinations were at the highest risk of experiencing injurious road accidents.

The prevalence for drug and alcohol found in this study is consistent with literature on impaired drivers, or drivers injured or killed in road accidents. However, prevalence figures are hard to compare because of time trends and local differences in drug use habits, patterns, and legislation (Morland, 2000). Our study confirms the well-known association between alcohol and traffic accidents (Borkenstein et al., 1974).

For drivers using benzodiazepines, a fivefold increased injury risk (95\% Cl: 1.8-14.0) was found, which is in concordance with other studies. In the late 70s, Skegg and co-workers linked drug prescription records with hospital admission and showed that people who used minor tranquillizers had a fivefold higher risk of a serious road accident (Skegg et al., 1979). A similar result was concluded in a related study, which found a four times increased risk for per-

Table 2

Risk for personal injury in road accidents associated with current use of psychoactive substances in real moving traffic

\begin{tabular}{lcl}
\hline Substance & $\mathrm{OR}^{\mathrm{a}}$ & $95 \% \mathrm{Cl}$ \\
\hline Amphetamines & 2.10 & $0.66-6.73$ \\
Benzodiazepines & 5.05 & $1.82-14.04$ \\
Cannabis & 1.22 & $0.55-2.73$ \\
Cocaine & 2.04 & $0.69-6.09$ \\
Opiates & 2.35 & $0.87-6.32$ \\
Blood alcohol concentration (g/l) & & \\
$\quad \quad 0.5$ & $1.00^{\mathrm{b}}$ & \\
$\quad 0.5-0.79$ & 5.46 & $1.28-23.22$ \\
$\quad \geq 0.8$ & 15.5 & $7.09-33.90$ \\
$\quad$ Multiple drugs vs. no drug & 6.05 & $2.60-14.10$ \\
Drug-alcohol combination vs. no drug & 112.22 & $14.10-893$ \\
\end{tabular}

Adjusted in the multivariate logistic model for age, gender, alcohol, amphetamines, benzodiazepines, cannabis, cocaine, opiates, season, and time of day.

${ }^{a} \mathrm{OR}$ : odds ratio.

${ }^{\mathrm{b}}$ Reference category. sons taking benzodiazepines within 4 weeks of receiving the prescription (Neutel, 1995) Also more recent data showed that users of benzodiazepines were at increased risk of experiencing road accidents (Barbone et al., 1998). In contrast, other studies using a comparable methodology showed no increase of accident risk with the use of benzodiazepines and sedatives (Jick et al., 1981; Leveille et al., 1994). However, Honkanen and others found a twofold increased accident risk among car drivers using benzodiazepines compared to controls. They selected controls randomly from car drivers at petrol stations, which comes closest to the methodology used in our study (Honkanen et al., 1980). Further research is necessary to establish whether within the group of benzodiazepines specific substances or doses bear a higher or lower risk than others due to differences in pharmacokinetic and pharmacodynamic properties.

The most common therapeutically used opioids studied in relation to traffic accidents are morphine and codeine. In our study, a twofold increased risk was found, which is in concordance with the literature (Ray et al., 1992; Leveille et al., 1994). We found a relatively high prevalence of opiates in injured drivers. After analytical confirmation 6-monoacetylmorphine (6-MAM) the first heroin metabolite was found in the urine of only one case, and two control patients. This figure may underestimate the real number of heroin users because 6-MAM is very rapidly metabolised into morphine and can therefore not always be detected.

The use of amphetamines (including ecstasy) and cocaine has been related with driving impairment (Dussault et al., 2001; Logan and Couper, 2001). In our study, we found that use of amphetamines or cocaine, and opiates places drivers at a twofold increased risk for motor vehicle accidents, however, not statistically significantly. The differences in the prevalence's of these drugs among drivers involved in an accident and non-accident drivers possibly indicates that these drugs are important risk factor for traffic accidents.

In our study, no association was found between exposure to cannabis and road accidents. Experimental and epidemiological studies have provided conflicting data about the 
role of cannabis to impair a driver's performance to that extent that it will comprise traffic accidents (Morland, 2000). However, the combination cannabis with alcohol is known to have serious effects on the driving performance (Robbe, 1998).

The potentials for bias must be considered as in every epidemiological study and the presented relative risks should be interpreted with caution. We sampled controls from moving traffic. Although the willingness to participate was high $(79 \%)$, the non-response may have led to selection bias. Theoretically, potential drug addicts might have refused to participate in our study. However, all motorists were by law compelled to undergo a breath test for alcohol. No major differences in age, sex, and alcohol levels were found when comparing controls who delivered a urine or blood specimen to motorists who did not. Thus, no indications of substantial selection bias in the control group were found.

The different distribution in the urine and blood specimens between the cases and controls may have led to information bias. The main strategy in our study was to get urine or blood as a biological sample and not to limit the inclusion of cases as well as controls by specifying one biological fluid. An advantage of urine over blood analysis is the longer persistence and higher concentrations of drugs and its metabolites (e.g. cannabis). However, this is also its major drawback as persistence for cannabis may vary from 1 day to several weeks after the pharmacological effect has disappeared. In that case, it is most unlikely that a positive drug result is related to an increased risk for road accidents. Because of the much larger share of urine specimens in the control group compared to the cases an underestimation of the relative risk for cannabis is possible. However, in this study it seems unlikely because overall $6.3 \%$ of all the urine specimens and $7.3 \%$ of all the blood specimens were positive for cannabis, respectively.

On the other hand, if the applied analytical method is not sensitive enough, drugs might escape detection. However, in our study this analytical bias is thought to be random; thus, there is no reason to believe that the level of error would differ between the study groups. Such bias will finally underestimate the study results (Hennekens and Buring, 1987).

Another limitation of this study is the sample size. The wide confidence intervals around some of the odds ratios are a reflection of this phenomenon. However, to our consideration expanding the number of cases would not change the main findings of our study. Expanding the sample size will decrease the confidence intervals around the estimates but will not majorly affect the point estimates in question.

The causal directions of the associations found in this study need to be considered. From a non-experimental study, it is difficult to unravel whether the drug (e.g. benzodiazepine), the reason for taking it or associated risk taking behaviour led to an increased accident risk (Neutel, 1995). Some underlying conditions, e.g. sleeping difficulties, may themselves lead to increased accident risks and become the causal factor itself, and not the benzodiazepine prescribed for this indication (Connor et al., 2002). However, there is substantial evidence from experimental placebo-controlled studies with patients previously diagnosed as insomniacs, that two nights of hypnotic drug use did cause a substantial impairing effect on real driving behaviour, whereas treatment significantly improved sleep quality (O'Hanlon and De Gier, 1986). Some benzodiazepine hypnotics caused effects in the morning after the second night and in the following afternoon that exceeded the effects of $0.5,0.8$ and $1.0 \mathrm{~g} / 1$ blood alcohol concentrations, which are used as legal limits in several countries around the world (Brookhuis, 1998). Also other risk taking behaviour, associated with drug use like overestimation of capabilities, macho attitude, habitual speeding, and other indecent driving behaviour, could be the causal factor (Petridou and Moustaki, 2000).

Generally, determining the relation between drug use and road trauma accidents is extremely complex because of many important selection factors that are doubtlessly present. Many reports have demonstrated a high rate of drug use among injured motorists, but few studies have collected comparable drug use data from a control group of motorists who were not involved in a traffic accident (Honkanen et al., 1980). Some researchers tried to overcome the control group problem by using a case-crossover design, as in the so-called culpability studies of (fatally) injured drivers (Robertson and Drummer, 1994; Lowenstein and Koziol-McLain, 2001). Important limitations of this type of studies are the generally small numbers of subjects, and subjective elements that are involved in evaluating culpability. Our study is an epidemiological study including a control group of non-accident drivers selected randomly from the moving traffic flow. The key advantage of this study is that the control (non-accident) drivers were legally stopped, tested for drug use and compared with a representative group of seriously injured drivers.

\section{Conclusions}

In conclusion, although a causal relationship between road trauma accidents and the use of drugs other than alcohol is not proven by the results of this study, it provides new insights in the role of these drugs in traffic crashes. Use of drug-drug combinations constitute a risk for drivers that will be at least as severe as the risk caused by the use of moderate quantities of alcohol. The impact on society is significant and actions related to reducing the use of these drugs by drivers are therefore needed. Still obvious gaps in our knowledge remain with respect to different effects of certain drugs within the same therapeutic class and drug combinations (multiple and with alcohol). Our study provides evidence that the use of drugs other than alcohol is associated with road traffic accidents. Users of these drugs should not drive a motor vehicle, especially not if taken in combination with alcohol. Legislators and health care providers will have to face this reality and act in 
accordance with new knowledge in order to prevent drugged driving.

\section{Acknowledgements}

The authors are indebted to R. Langen and F. van Hoof for their critical comments regarding the laboratory drug analysis. We acknowledge The Netherlands Forensic Institute, the Dutch Laboratory for Drugs Doping and the SWOV Institute for Road Safety Research for their co-operation in this study.

\section{References}

Baker, S.P., O’Neill, B., Haddon Jr., W., Long, W.B., 1974. The injury severity score: a method for describing patients with multiple injuries and evaluating emergency care. J. Trauma 14, 187-196.

Barbone, F., McMahon, A.D., Davey, P.G., Morris, A.D., Reid, I.C., McDevitt, D.G., MacDonald, T.M., 1998. Association of road-traffic accidents with benzodiazepine use. Lancet 352, 1331-1336.

Borkenstein, R.F., Crowther, R.F., Shumate, R.P., Ziel, W.B., Zylman, R., 1974. The role of the drinking driver in traffic accidents (the Grand Rapids Study). Blutalcohol 11 (Suppl. 1), 7-13.

Brookhuis, K.A., 1998. How to measure driving ability under influence of alcohol and drugs, and why. Hum. Psychopharmacol. 13, S64-S69.

Connor, J., Norton, R., Ameratunga, S., Robinson, E., Civil, I., Dunn, R., Bailey, J., Jackson, R., 2002. Driver sleepiness and risk of serious injury to car occupants: population based case control study. Br. Med. J. 324, 1125.

Dussault, C., Brault, M., Lemire, A.M., Bouchard, J., 2001. The Role of cocaine in fatal crashes: first results of the Quebec drug study. Proceedings of the Annual Conference for the Advancement of Automotive Medicine, 45, 125-137.

Gjerde, H., Beylich, K.M., Morland, J., 1993. Incidence of alcohol and drugs in fatally injured car drivers in Norway. Accid. Anal. Prev. 25, $479-483$.

Hemmelgarn, B., Suissa, S., Huang, A., Boivin, J.F., Pinard, G., 1997. Benzodiazepine use and the risk of motor vehicle crash in the elderly. J. Am. Med. Assoc. 278, 27-31.

Hennekens, C.H., Buring, J.E., 1987. Epidemiology in Medicine. Little, Brown and Company, Boston.

Honkanen, R., Ertama, L., Linnoila, M., Alha, A., Lukkari, I., Karlsson, M., Kiviluoto, O., Puro, M., 1980. Role of drugs in traffic accidents. Br. Med. J. 281, 1309-1312.

Hosmer, D.W., Lemeshow, S., 1989. Applied Logistic Regression. Wiley, New York.

Jick, H., Hunter, J.R., Dinan, B.J., Madsen, S., Stergachis, A., 1981. Sedating drugs and automobile accidents leading to hospitalization. Am. J. Public Health 71, 1399-1400.
Leveille, S.G., Buchner, D.M., Koepsell, T.D., McCloskey, L.W., Wolf, M.E., Wagner, E.H., 1994. Psychoactive medications and injurious motor vehicle collisions involving older drivers. Epidemiology 5, 591598

Logan, B.K., Couper, F.J., 2001. 3,4-Methylenedioxymethamphetamine (MDMA, ecstasy) and driving impairment. J. Forensic Sci. 46, 14261433.

Lowenstein, S.R., Koziol-McLain, J., 2001. Drugs and traffic crash responsibility: a study of injured motorists in Colorado. J. Trauma 50, 313-320.

Marquet, P., Delpla, P.A., Kerguelen, S., Bremond, J., Facy, F., Garnier, M., Guery, B., Lhermitte, M., Mathe, D., Pelissier, A.L., Renaudeau, C., Vest, P., Seguela, J.P., 1998. Prevalence of drugs of abuse in urine of drivers involved in road accidents in France: a collaborative study. J. Forensic Sci. 43, 806-811.

McGwin Jr., G., Sims, R.V., Pulley, L., Roseman, J.M., 2000. Relations among chronic medical conditions, medications, and automobile crashes in the elderly: a population-based case-control study. Am. J. Epidemiol. 152, 424-431.

Morland, J., 2000. Driving under the influence of non-alcohol drugs. Forensic Sci. Rev. 12, 80-105.

Neutel, C.I., 1995. Risk of traffic accident injury after a prescription for a benzodiazepine. Ann. Epidemiol. 5, 239-244.

O'Hanlon, J.F., De Gier, J.J., 1986. Drugs and Driving. Taylor \& Francis, London, PA.

O'Hanlon, J.F., Volkerts, E.R., 1986. Hypnotics and actual driving performance. Acta Psychiatr. Scand. 332, 95-104.

Petridou, E., Moustaki, M., 2000. Human factors in the causation of road traffic crashes. European J. Epidemiol. 16, 819-826.

Ramaekers, J.G., 1998. Behavioural toxicity of medicinal drugs. Practical consequences, incidence, management and avoidance. Drug Safety 18 , 189-208.

Ray, W.A., Fought, R.L., Decker, M.D., 1992. Psychoactive drugs and the risk of injurious motor vehicle crashes in elderly drivers. Am. J. Epidemiol. 36, 873-883.

Robbe, H., 1998. Marijuana's impairing effects on driving are moderate when taken alone but severe when combined with alcohol. Hum. Psychopharmacol. 13, S70-S78.

Robertson, M.D., Drummer, O.H., 1994. Responsibility analysis: a methodology to study the effects of drugs in driving. Accid. Anal. Prevent. 26, 243-247.

Skegg, D., Richards, S., Doll, R., 1979. Minor tranquillisers and road accidents. Br. Med. J. 1, 917-919.

Smiley, A., 1987. Effects of minor tranquilizers and antidepressants on psychomotor performance. J. Clin. Psychiatry 48, 22-28.

Soderstrom, C.A., Smith, G.S., Dischinger, P.C., McDuff, D.R., Hebel, J.R., Gorelick, D.A., Kerns, T.J., Ho, S.M., Read, K.M., 1997. Psychoactive substance use disorders among seriously injured trauma center patients. J. Am. Med. Assoc. 277, 1769-1774.

Vles, W.J., Kroezen, F., Meeuwis, J.D., Leenen, L.P.H., 2000. Trauma registration in a Dutch Trauma population with emphasis on quality of care. European J. Trauma 26, 248-255. 